
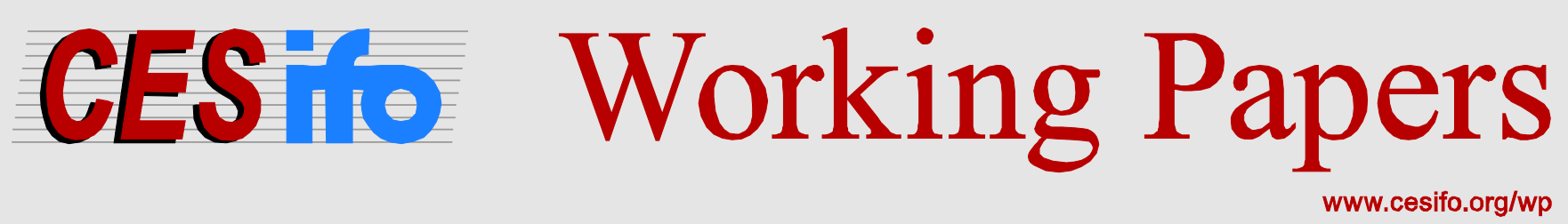

\title{
Dispersed Information and the Origins of Aggregate Fluctuations
}

\author{
Jakob Grazzini \\ Domenico Massaro
}

\author{
CESIFO WORKING PAPER NO. 5957 \\ CATEgory 6: FisCAl POLICY, MaCROECONOMICS AND GROWTH \\ JUNE 2016
}

An electronic version of the paper may be downloaded

- from the SSRN website:

- from the RePEc website:

- from the CESifo website:

www.SSRN.com

www.RePEc.org

www.CESifo-group.org/wp 


\title{
Dispersed Information and the Origins of Aggregate Fluctuations
}

\begin{abstract}
In the presence of dispersed information, agents may decide to take into account the actions of other agents because of the possible additional information conveyed by these actions. We call the act of using other agents' actions in the individual decision process social learning. This paper argues that social learning aimed at increasing the precision of individual information may lead to aggregate fluctuations. We consider a setting where firms receive independent noisy signals about a common fundamental and can observe other firms' actions through a network of informational links. We show that, when firms can observe each other's decisions, they are able to increase the accuracy of their actions. While reducing volatility at the individual level, social learning may lead to an increase in volatility at the aggregate level depending on the network topology. Moreover, if the network is very asymmetric, aggregate volatility does not decay as predicted by the law of large numbers.
\end{abstract}

JEL-Codes: D830, D850, E320.

Keywords: business cycle, social networks, dispersed information, aggregate volatility.

\author{
Jakob Grazzini \\ Catholic University of Milan \\ Department of Economics and Finance \\ \& Complexity Lab in Economics \\ Milan / Italy \\ jakob.grazzini@unicatt.it
}

\author{
Domenico Massaro \\ Catholic University of Milan \\ Department of Economics and Finance \\ \& Complexity Lab in Economics \\ Milan / Italy \\ domenico.massaro@unicatt.it
}

June 8, 2016

We thank Carlo Lucibello, Nicolò Pecora, Paolo Pin and the participants to the CESifo Area Conference on Macro, Money and International Finance (Munich, 2016), JAFEE20 International Conference (Tokyo, 2016) for stimulating discussions and helpful comments. Jakob Grazzini acknowledges financial support from the European Union, Seventh Framework Programme FP7/2007-2013 Socio-economic Sciences and Humanities under Grant Agreement No. 612796 - MACFINROBODS. Domenico Massaro acknowledges financial support from the Ministry of Education, Universities and Research of Italy (MIUR), program SIR (grant n. RBSI144KWH). None of the above are responsible for errors in this paper. 


\section{Introduction}

Human beings are social animals. This simple truth is often neglected when analysing human behavior in macroeconomics. The characteristic of social animals is that they live in groups and interact with other members of the group to perform vital tasks, such as defence or feeding. Several studies in biology document forms of interaction in which the group allows individuals to take advantage of the information gathered by others. Pulliam (1973) for example studies the flocking behavior of finches and shows that an advantage of feeding in groups is to increase the probability to detect a predator ("many eyes effect"). If one finch spots a predator and decides to fly off in alarm, the other finches observe this action and follow without having actually seen the danger. Moreover, assuming that anti-predatory vigilance is costly, for example because it is time consuming and alternative to feeding, the group provides a simple and effective cost sharing mechanism (Fernandez et al., 2003). In fact, the propensity to live in groups and learn from others' behavior can be considered an evolutionary response that promotes survival in complex environments for both animals and human beings (Henrich, 2015). Several experiments in psychology (e.g., Asch, 1961) and economics (e.g., Apesteguia et al., 2007; Lahno and Serra-Garcia, 2015) document indeed that the behavior of peers influences decision making under many different circumstances.

There are in fact countless social and economic situations in which human beings are influenced by what others around them are doing when deciding upon an action. The decisions of others can be relevant for individual decision making for a variety of reasons. First, in the presence of payoff externalities, the actions of others can directly influence the utility function. An example is the adoption of new information technologies where individuals decide to adopt technologies, e.g., operating systems, whose value depends on the adoption choices of others. The actions of other agents may matter also because of "social pressure", i.e., the influence exerted by a group on the behavior of individuals seeking social conformity (or social nonconformity). The relevance of social conformity in economics was already recognized by J.M. Keynes, who argues that investment decisions can be biased by the incentive of reputation: "Worldly wisdom teaches that it is better for reputation to fail conventionally than to succeed unconventionally" (Keynes, 1936, p. 158). Finally, the decisions of other agents may matter for individual decision making when such decisions are perceived as reflecting relevant information. In a complex world, it is difficult to collect and process 
all the available information. Observing the actions of others can allow to exploit the information - and the processing capability - available to other members of the group. This is the scenario we analyze in this paper and, following Bandura and McClelland (1977), we call the act of observing and learning from other agents' actions social learning.

The goal of this paper is to investigate the consequences of social learning for aggregate outcomes and to study how aggregate volatility depends on the structure of the social network shaping the patterns of interaction and learning among individuals. To this end, we consider a setting where agents receive independent noisy signals about the true value of a common variable of interest and social learning occurs through an arbitrary social network. Although in our setting individual payoffs do not depend on the decisions of others, paying attention to other individuals is rational because their decisions may reflect valuable additional information. This simple setup, in which there are no strategic complementarities and the interaction among agents is purely informational, allows us to isolate the impact of different network topologies on aggregate fluctuations. ${ }^{1}$

The essence of the model is as follows. A set of agents must take an action, receive a private signal on a common payoff-relevant fundamental state variable, and can observe the actions of a subset of other agents. The subset of observable agents is defined by a social network. The network is represented as a weighted directed graph indicating whether agent $i$ can observe the action of agent $j$ (informational link), and how much weight the agent gives to such decision. The "observational structure" defined by the network is exogenous and can correspond to e.g., geographical proximity or social relationships.

For the sake of concreteness, we frame the analysis in a setup where firms must decide the investment level before they can observe the true return on capital. They receive a private signal on the common productivity and they can observe the investment decisions of a subset of other firms. In this setting we show that social learning aimed at increasing the precision of individual information may lead to aggregate fluctuations. Our main results can be summarised as follows. The case of isolated firms, i.e., firms acting only in reaction to their own signal, represents an upper bound for the volatility of individual investment decision. In fact, when firms can observe

\footnotetext{
${ }^{1}$ Models with noisy signals and strategic complementarities have been considered by Morris and Shin (2002), Angeletos and Pavan (2007), Angeletos and La'O (2013), Colombo et al. (2014), Benhabib et al. (2015), Chahrour and Gaballo (2015) and Angeletos et al. (2016) among others. All papers mentioned above abstract from considerations about the macroeconomic impact of social learning for different social networks topologies, which is instead the focus of this paper.
} 
each other's decisions, they are able to increase the accuracy of their investment plans. This is quite intuitive as each firm is able to exploit the additional information embedded in other firms' decisions. Moreover, this result is independent on the topology of the network.

While reducing volatility at the individual level, social learning may, on the other hand, lead to an increase in volatility at the aggregate level. In fact, the case of isolated firms represents a lower bound for the volatility of aggregate investment and, according to the properties of the network topology, learning by observing other firms' behavior can imply a higher variance of aggregate investment.

Our paper also contributes to the debate on whether aggregate volatility can originate from fluctuations at the micro level. Following the standard diversification argument, in an economy composed by $N$ firms, aggregate volatility should decay at a rate $1 / \sqrt{N}$ (see e.g., Lucas, 1977). We show that the diversification argument may not hold in the presence of dispersed information and social learning and that social networks may translate imperfect information into volatility at the aggregate level. In particular, our results demonstrate that when the informational network is very asymmetric, then aggregate volatility decays at a rate much slower than $1 / \sqrt{N}$. In other words when many firms look at the decision of the same small number of firms, then the influence exerted by firms that are very central in the informational network does not decay as the number of firms in the economy increases.

Our work relates to several strands of research. Banerjee (1992), Bikhchandani et al. (1992) and Smith and Sørensen (2000) among others study learning models in which agents can observe the actions of other agents. These papers investigate whether sequential learning mechanisms, defined as observational learning, lead to informational cascades, and to an inefficient aggregation of private information. Informational cascades are defined in Bikhchandani et al. (1992) as situations in which agents follow the actions of the preceding agents, disregarding their own private information. Informational cascades emerge when the set of possible actions is discrete (Bikhchandani et al., 1998). In our analysis we consider simultaneous decisions as in Gale and Kariv (2003), and a continuous set of possible actions, ruling out the possibility of informational cascades. Moreover, we introduce a network structure defining the patterns of social learning and show that it plays an important role for aggregate outcomes. Ellison and Fudenberg $(1993,1995)$ study private information aggregation when agents can observe choices and payoffs of other agents and use rule of thumb 
heuristics to decide their own action. They show that even with simple heuristic behaviors, social learning can lead to efficient outcomes. We analyze instead a framework in which agents do not observe other agents' payoffs and focus on the impact of the observational network on individual and aggregate volatility.

The papers stemming from the seminal contribution of DeGroot (1974), e.g., Bala and Goyal (1998), Golub and Jackson (2010), Acemoglu et al. (2011) and DeMarzo et al. (2003), analyze instead richer network structures. These papers focus on network topologies ensuring convergence to the true underlying fundamental (or unidimensional opinions) both under Bayesian and nonBayesian learning/updating of beliefs. We consider a different mechanism of beliefs' updating as described in Section 2 and focus on aggregate volatility.

Another important stream of literature related to our work concerns the social value of public information in presence of imperfect private information (see e.g., Morris and Shin, 2002; Angeletos and Pavan, 2004; Colombo et al., 2014, among others). In particular, the model in which we frame our analysis is similar to the model described in Angeletos and Pavan (2004), but with several substantial differences. First, the focus of our paper is on the impact of social learning on aggregate volatility rather then on social welfare. Second, in our framework agents do not have access to public information, but they can observe the actions of different subsets of other agents. Finally, to isolate the network effect on the individual actions, we assume that the agents do not have a beauty contest incentive. ${ }^{2}$

Finally, our work is also related to the literature on the role of idiosyncratic shocks at the micro level in macroeconomic fluctuations. Dupor (1999) and Horvath (1998, 2000) debated about the diversification argument mentioned above. Gabaix (2011) shows that the $1 / \sqrt{N}$ diversification argument does not apply when the firm size distribution is sufficiently fat-tailed, while Acemoglu et al. (2012) show that the argument is not valid in the presence of asymmetric input-output links between sectors. ${ }^{3}$ We show that, even in the presence of firms with identical size and without inputoutput relations between different sectors, the diversification argument may fail in the presence of dispersed information and social learning.

\footnotetext{
${ }^{2}$ In the terms of the model described in Angeletos and Pavan (2004), we assume that the individual return to investment does not depend on the aggregate level of investment.

${ }^{3}$ Earlier contributions on the topic include Jovanovic (1987) and Durlauf (1993) who show that strategic complementarities and local firms' interactions may translate shocks occurring at the firm-level into aggregate volatility. Moreover, Bak et al. (1993) focus on the role of supply chains in aggregate fluctuations.
} 
The outline of the paper is as follows. Section 2 presents a model with dispersed information and social learning. Section 3 analyzes the effect of different network topologies on volatility at the individual and aggregate level. Section 4 discusses social learning in a Lucas-Phelps island model and shows the effect of dispersed information and social learning on aggregate GDP. While the previous sections derive results for a general social learning mechanism in which weights on different sources of information can be set arbitrarily, Section 5 analyzes the case in which agents set these weights optimally. Section 6 concludes.

\section{Model}

The economy is populated by $N$ firms indexed by $i=1 \ldots N$. Preferences and technologies are modeled as in Angeletos and Pavan (2004). In particular, firms are risk-neutral with utility

$$
u_{i}=\theta k_{i}-\frac{1}{2} k_{i}^{2}
$$

where $\theta \in \mathbb{R}$ is the return to investment, $k_{i} \in \mathbb{R}$ is the investment decision and $k_{i}^{2} / 2$ is the cost of investment. The exogenous random variable $\theta$ parameterizes the fundamentals of the economy and is assumed to be drawn from an improper uniform distribution (Morris and Shin, 2002).

Differently from Angeletos and Pavan (2004), in our framework the return on capital does not depend on the investment decision of the other agents in the economy. This implies that there is no complementarity between investment decisions. The fundamental $\theta$ is unknown when investment decisions are made so that each agent chooses $k_{i}$ to maximize $\mathrm{E}_{i}\left[u_{i}\right]$. This implies that the optimal investment of firm $i$ is

$$
k_{i}=\mathrm{E}_{i}[\theta]=\mathrm{E}\left[\theta \mid \mathcal{I}_{i}\right]
$$

where $\mathcal{I}_{i}$ is the information set available to agent $i$. Firms receive a private signal $s_{i}$ on the fundamental

$$
s_{i}=\theta+\sigma \varepsilon_{i},
$$

where $\sigma$ is the standard deviation of the private signal and $\varepsilon_{i} \sim \mathcal{N}(0,1)$ is an i.i.d. disturbance. Eqs. (2) - (3) ensure that firms' payoffs are independent from each other and that interaction among 
firms is purely informational.

When firms cannot observe investment decisions of other firms in the economy, the information set of each firm consists only of the private signal. Therefore in isolation we have that

$$
k_{i}=\mathrm{E}\left[\theta \mid \mathcal{I}_{i}\right]=s_{i}
$$

Now consider the case in which firms can observe the actions of a subset of other firms (as in e.g., Banerjee, 1992; Bikhchandani et al., 1992). ${ }^{4}$ If firms have access to information about the behavior of other firms, i.e., to their investment decisions, they will rationally use this information in order to average out the idiosyncratic noise. The rationality argument follows from the fact that the signal $s$ is an unbiased predictor of the fundamental $\theta$ and from the fact that firms take optimal decisions given their information. This implies that $k_{i}$ is an unbiased predictor of $\theta$. The firms' interaction network is described by an $N \times N$ non-negative matrix $\mathrm{W}$, where each element $w_{i j}>0$ indicates an informational link from firm $i$ to firm $j$, i.e., firm $i$ pays attention to the decision of firm $j$. The matrix $\mathrm{W}$ can be asymmetric and informational links can be one-sided, so that $w_{i j}>0$ and $w_{j i}=0$. The network topology determines the subset of other firms observable by each firm. If firm $i$ can observe firm $j$, the weight $w_{i j}>0$, otherwise $w_{i j}=0$. Moreover, we assume that the elements on the main diagonal of $\mathrm{W}$ are zeros, i.e., $w_{i i}=0$, meaning that, quite naturally, firms do not need to form an informational link with themselves. The specific value of $w_{i j}>0$ can be interpreted as the relative precision of information about the fundamentals reflected in the investment decision of firm $j$, as perceived by firm $i$ (see Golub and Jackson, 2010). ${ }^{5}$ Matrix $\mathrm{W}$ is stochastic so that its entries across each row are normalized to sum to 1 , i.e., $\sum_{j} w_{i j}=1$. The information provided by the network on the fundamentals is therefore a weighted average of observed firms' decisions, i.e., $\sum_{j} w_{i j} k_{j}$.

Based on both private information and information coming from the network, firm $i$ 's expected

\footnotetext{
${ }^{4}$ In appendix B we show that results described below do not change if we assume that the agents can observe the private signals of other agents, rather than the actions.

${ }^{5}$ Notice that we allow firm $i$ 's assessment of the precision reflected in firm $j$ 's decision to be subjective for the sake of generality, but it could also be objectively correct for all firms.
} 
value of $\theta$ and optimal investment decision is given by:

$$
k_{i}=\alpha_{i} s_{i}+\left(1-\alpha_{i}\right) \sum_{j} w_{i j} k_{j}
$$

where the parameter $\alpha_{i}$ measures the importance imputed by firm $i$ to its signal relative to the information conveyed by the network. In principle the weights $\left\{\alpha_{i}, w_{i j}\right\}$ on the different sources of information could be set optimally by firms, but perfectly rational aggregation of information requires taking into account the exact structure of the network. In fact, the precision of the information conveyed by the investment decision of each firm $j$, depends not only on the precision of its signal $s_{j}$, but also on the precision of the information conveyed by its observational network, i.e., on the information contained in the investment decisions of other firms observed by firm $j$ and so on. This is an especially complex problem because firms connected through a network may receive information both directly and indirectly from the same source. Therefore, in order to optimally weight the information received, firms would need to know the source of all the information that influenced, both directly and indirectly, the decisions of other firms. DeMarzo et al. (2003) argue that agents may have difficulties with the weighting process and propose a model in which agents are subject to persuasion bias, i.e., they fail to properly account for possible repetitions of the information they receive. Brandts et al. (2015) present experimental evidence consistent with the model of persuasion bias of DeMarzo et al. (2003).

Given that, in the light of the previous considerations, fully Bayesian learning in this setup appears to be too complicated to be realistic, in our analysis we consider generic values of the weights $w_{i j}$ and $\alpha_{i}$. Eq. (5) can be interpreted as a general heuristic to deal with the complex inference problem at hand, where the weights $w_{i j}$ are a subjective evaluation of the relative precision of the decisions of observed firms, and $\alpha_{i}$ is a subjective evaluation of the relative precision of private information and the information obtained from the network. We remark nevertheless that this is a general framework that nests the optimally chosen weights as a special case, which will be analyzed in Section 5. 


\section{Timing and equilibrium}

In each period firms choose actions simultaneously. Within the same period, each firm $i$ observes the actions chosen by other firms in the social network and updates its beliefs according to Eq. (5). Let $k$ denote the $N \times 1$ vector of individual investment decisions, $s$ the $N \times 1$ vector of private signals, and $\mathrm{D}$ the diagonal matrix such that $[\mathrm{D}]_{i i}=\alpha_{i}, \forall i \in[1, N]$. We can then write Eq. (5) in vectorial form as

$$
k=\mathrm{D} s+(\mathrm{I}-\mathrm{D}) \mathrm{W} k
$$

where the entries of matrices D and $\mathrm{W}$ are fixed and depend on the exogenous network structure and on the (perceived) precision of different sources of information. Equilibrium in each period is reached as the fixed point of the dynamic process

$$
k_{\tau}=\mathrm{D} s+(\mathrm{I}-\mathrm{D}) \mathrm{W} k_{\tau-1}
$$

occurring in notional time $\tau$. Before identifying conditions for the existence of the fixed point of the dynamic process above, let us introduce the following definitions. Following Golub and Jackson (2010), we define a group of nodes $Z \subset N$ as closed relative to a generic adjacency matrix $\Omega$ if $i \in Z$ and $\omega_{i j}>0$ imply that $j \in Z$. A closed group of nodes is minimally closed relative to $\Omega$ if it is closed and no nonempty strict subset is closed.

The following lemma establishes the convergence result for the dynamic process in Eq. (7).

Lemma 1. The matrix difference equation (7) converges to

$$
k=[\mathrm{I}-(\mathrm{I}-\mathrm{D}) \mathrm{W}]^{-1} \mathrm{D} s
$$

if one of the following conditions is satisfied

a) $0<\alpha_{i} \leq 1 \forall i \in[1, N]$ for any network topology.

b) $0 \leq \alpha_{i} \leq 1$ and for each minimally closed group $\mathcal{Z}_{j}$ relative to $(\mathrm{I}-\mathrm{D}) \mathrm{W}$ there exists at least one $i \in \mathcal{Z}_{j}$ such that $\alpha_{i}>0$.

Intuitively, Lemma 1 states that for equilibrium in Eq. (8) to exist, each firm in the network 
must be reached directly or indirectly by at least one signal. In case a) this condition is obviously satisfied since each firm gives a positive weight to its private signal. In case b) we consider the case in which $\alpha_{i}=0$ for some firms. According to Lemma 1, for the equilibrium to exist there must be at least one $\alpha_{i}>0$ in each minimally closed group. This implies that all firms in each minimally closed group, and all firms connected directly or indirectly to any minimally closed group, are reached by at least one signal.

The equilibrium in Eq. (8) is reminiscent of the equilibrium in Acemoglu et al. (2012) but with the following important differences. Firstly, Eq. (8) is derived in a dispersed information setup rather than an input-output multisectoral model, leading to different interpretations of structural parameters and economic results. Secondly, while Acemoglu et al. (2012) consider the case of homogeneous $\alpha$, representing the labor share in the production technology, we analyze the case of heterogeneous $\alpha_{i}$, representing individual information weighting.

At this point it is also worth emphasizing the differences between the belief updating mechanism à la DeGroot (1974) (see e.g., DeMarzo et al., 2003; Golub and Jackson, 2010, among others) and the process in Eq. (7). While in DeGroot (1974) agents' beliefs are in each round of updating given by a weighted average of previous period's beliefs, in Eq. (7) we allow agents to bias their decisions towards their private signal. Therefore, when $\alpha_{i}>0$, the equilibrium decision of firm $i$ always includes information from its private signal. The updating mechanism à la DeGroot is nested in formulation (7) and can be recovered by setting $\alpha_{i}=0$ and $w_{i i} \geq 0 \forall i$. In this case Golub and Jackson (2010) show that limiting beliefs may converge to solutions, periodic or aperiodic, in which agents do not take into account their private signal in equilibrium.

In what follows we will consider the case in which $0<\alpha_{i} \leq 1 \forall i \in[1, N]$, firstly because it seems reasonable that firms always place some weight on their private signals, and secondly because in this case the equilibrium in Eq. (8) always exists for any network configuration. This allows us to derive general results about the impact of different observational topologies on individual and aggregate volatility. Nevertheless, we remark that our conclusions also apply to the cases in which $0 \leq \alpha_{i} \leq 1$ stated in Lemma 1 point $\left.\mathrm{b}\right)$.

Eq. (8) shows that, when firms' actions are observable through the informational network, in equilibrium the individual investment decisions are a linear combination of the private signals. The extreme case in which $\alpha_{i}=1 \forall i$ is equivalent to the case of isolation in which firms only consider 
their private information and the network does not play any role.

Before proceeding to the analysis of the impact of social learning on volatility, we define aggregate investment as the sum of individual investment decisions, normalized by the number of agents in the economic system:

$$
K=\frac{1}{N} \sum_{i} k_{i} .
$$

In the following section we present the main results of the paper, namely that in the presence of dispersed information, social learning increases the precision of firms' investment decisions (reducing therefore the variance of individual investments with respect to isolation), and that at the same time the social learning process leads to increased volatility at the aggregate level (increasing therefore the variance of aggregate investment with respect to isolation) depending on the topology of the informational network. Moreover, we also characterize the decay of aggregate volatility as the number of firms $N \rightarrow \infty$ and show that social learning may lead to a decay rate slower than $1 / \sqrt{N}$ depending on the network topology.

\section{$3 \quad$ Network Topology and Micro/Macro Volatility}

In this section we focus on the impact of social learning on both micro and macro volatility, and relate it to the structure of the network characterizing the interaction patterns among firms.

To help the intuition we will illustrate our results using simple examples. Fig. 1 displays two different configurations of the economy. In both cases we have $N=5$ and all $N$ firms observe the actions of another firm in the economy. In the economy depicted in Fig. 1(a) as a directed star network, all firms observe the same firm $i=1$ and the latter observes another firm $j=2$. In the economy depicted in Fig. 1(b) as a directed regular network, each firm observes the investment decision of a different firm.

The corresponding adjacency matrices representing the star and the regular networks are shown in Eq. (10).

$$
\mathrm{W}_{\text {star }}=\left(\begin{array}{ccccc}
0 & 1 & 0 & 0 & 0 \\
1 & 0 & 0 & 0 & 0 \\
1 & 0 & 0 & 0 & 0 \\
1 & 0 & 0 & 0 & 0 \\
1 & 0 & 0 & 0 & 0
\end{array}\right) \quad \mathrm{W}_{\text {regular }}=\left(\begin{array}{ccccc}
0 & 1 & 0 & 0 & 0 \\
0 & 0 & 1 & 0 & 0 \\
0 & 0 & 0 & 1 & 0 \\
0 & 0 & 0 & 0 & 1 \\
1 & 0 & 0 & 0 & 0
\end{array}\right)
$$




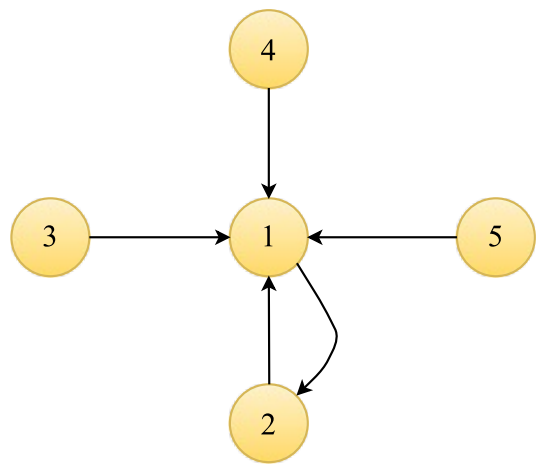

(a) Star network.

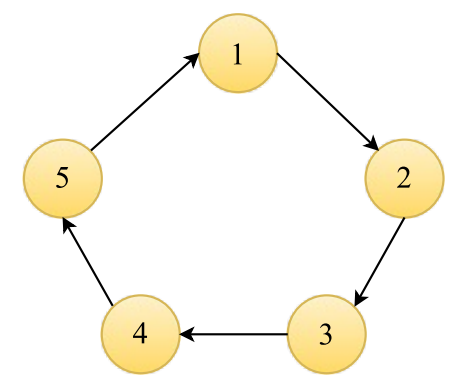

(b) Regular network.

Figure 1: Different configurations of the economy.

In both cases, given that each firm can observe only another firm in the economy, the weight assigned to that observation is equal to 1 by construction. In the following sections we will use these simple network configurations as examples to illustrate the impact of different topologies on the micro and macro properties of the economic system.

\subsection{Volatility of Individual Investment}

In what follows we show that incorporating information from other firms in investment decisions is rational from a generic firm i's point of view, as social learning leads to lower variance of individual investment. Using Eq. (8), individual investment in equilibrium can be written as

$$
k_{i}=\sum_{j} \hat{w}_{i j} \alpha_{j} s_{j}
$$

where $\hat{w}_{i j}$ denotes the element $(i, j)$ of the matrix $\hat{\mathrm{W}}$ defined as $\hat{\mathrm{W}} \equiv[\mathrm{I}-(\mathrm{I}-\mathrm{D}) \mathrm{W}]^{-1}$. The variance of $k_{i}$ is then given by

$$
\operatorname{var}\left(k_{i}\right)=\sum_{j} \hat{w}_{i j}^{2} \alpha_{j}^{2} \sigma^{2}
$$

In the case of isolated firms there are no informational links between firms, i.e., $\mathrm{W}$ is a matrix of zeros (hence $\hat{\mathrm{W}}$ is an identity matrix), and $\alpha_{i}=1 \forall i$, meaning that firms only consider their private signal and therefore

$$
\operatorname{var}\left(k_{i}\right)=\sigma^{2}
$$


Social learning happens when $\alpha_{i}<1$ and $w_{i j}>0$ for at least one $j \neq i$, which means that firm $i$ has at least one informational link with another firm $j$ in the economy, and uses the information embedded in the decision made by firm $j$. The impact of social learning on individual volatility is stated in the following proposition:

Proposition 1. The variance of individual investment in the case of social learning is always less than the variance of individual investment in case of isolated firms, that is

$$
\sum_{j} \hat{w}_{i j}^{2} \alpha_{j}^{2}<1
$$

The proof is in Appendix A. Proposition 1 shows that social learning is rational from the firm point of view. In this way firms are in fact able to increase the precision of their individual forecasts on the fundamental. The intuition for this result is that the information about fundamentals contained in individual signals is spread through the network and in equilibrium firms are able to exploit this additional information by observing the behavior of other firms.

The following example illustrates the information spreading mechanism.

\section{Example 1.}

Consider an economy with $N=5$ firms. In the absence of informational links we have that each firm sets $k_{i}$ in reaction to its own signal only, i.e., $k_{i}=s_{i}$, and therefore the variance of individual investment is given by $\sigma^{2}$ defined as before.

Suppose instead that the informational structure of the economy is described by the regular network in Fig. 1(b). In this case the investment decision of e.g., firm 1 is taken according to $k_{1}=\alpha_{1} s_{1}+\left(1-\alpha_{1}\right) w_{12} k_{2}$, i.e., using both the private signal $s_{1}$ and the decision of firm 2, which in turn is taken using its private signal $s_{2}$ and the decision of firm 3, and so on. The network structure allows to incorporate information from other firms' signals in individual decisions. In fact, in equilibrium we have that production decisions are determined according to Eq. (8) and therefore the way in which private signals are spread through the network depends on the matrix $C \equiv[I-(I-D) W]^{-1} D$. The specific value of $0<\alpha_{i} \leq 1$, contained in matrix $D$, does not influence the ability of the network to spread the information. For the sake of illustration we assume in this 
example that $\alpha_{i}=\alpha=0.5 \forall i$ without loss of generality. In Section 5 we analyze the same type of economy when firms set weights optimally and show that the qualitative conclusions do not change. Given the assumption on the observational network and on $\alpha$, it is easy to compute the matrix $\mathrm{C}_{\text {regular }} \equiv\left[\mathrm{I}-(\mathrm{I}-\mathrm{D}) \mathrm{W}_{\text {regular }}\right]^{-1} \mathrm{D}$ :

$$
\mathrm{C}_{\text {regular }}=\left(\begin{array}{lllll}
0.5161 & 0.2581 & 0.1290 & 0.0645 & 0.0323 \\
0.0323 & 0.5161 & 0.2581 & 0.1290 & 0.0645 \\
0.0645 & 0.0323 & 0.5161 & 0.2581 & 0.1290 \\
0.1290 & 0.0645 & 0.0323 & 0.5161 & 0.2581 \\
0.2581 & 0.1290 & 0.0645 & 0.0323 & 0.5161
\end{array}\right)
$$

The expression above shows that, due to the network of observational links, the individual investment decision of each firm is influenced by the private signals of all firms in the economy. For example, the decision $k_{1}$ of firm 1 is given by

$$
k_{1}=\sum_{j=1}^{5} c_{1 j} s_{j}=0.5161 s_{1}+0.2581 s_{2}+0.1290 s_{3}+0.0645 s_{4}+0.0323 s_{5}
$$

Given the topology of the regular network we have that, for each firm, the variance of individual investment with social learning is given by $0.09 \sigma^{2}$ and thus smaller than the case of isolation. Social learning allows to exploit the information contained in individual actions and thus reduces the errors in individual investment decisions.

The reduction in individual volatility in the presence of social learning with respect to the case of isolation is independent on the network structure. In fact, as long as a firm has at least one informational link and therefore looks at the decision of at least one additional firm (on top of reacting to its own signal), the variance of its investment will be lower than the case of isolation.

Nevertheless, different network topologies may imply different levels of individual volatility, as shown in the following example.

\section{Example 2.}

Consider an economy with an informational structure described by the star network in 
Fig. 1(a) and keep the assumption $\alpha_{i}=\alpha=0.5 \forall i$. In this case we have that

$$
\mathrm{C}_{\text {star }}=\left(\begin{array}{ccccc}
0.67 & 0.33 & 0 & 0 & 0 \\
0.33 & 0.67 & 0 & 0 & 0 \\
0.33 & 0.17 & 0.50 & 0 & 0 \\
0.33 & 0.17 & 0 & 0.50 & 0 \\
0.33 & 0.17 & 0 & 0 & 0.50
\end{array}\right)
$$

The expression above shows that the decisions of firms 3, 4 and 5 are based on information contained in the individual signals of two additional firms, while the decisions of firms 1 and 2 only exploit information from one additional firm. We have therefore that $\operatorname{var}\left(k_{1}\right)=\operatorname{var}\left(k_{2}\right)=0.14 \sigma^{2}$, while $\operatorname{var}\left(k_{3}\right)=\operatorname{var}\left(k_{4}\right)=\operatorname{var}\left(k_{5}\right)=0.10 \sigma^{2}$, meaning that individual variances are higher than in the case of a regular network where each individual decision exploits information from all the firms in the economy, but still lower than in the case of isolated firms.

\subsection{Volatility of Aggregate Investment}

In this section we show that when firms look at other firms' decisions to make their own investment plans, the aggregate variance may increase with respect to the case of isolation depending on the topology of the informational network. We denote aggregate investment normalized by the number of agents in the economy as

$$
\begin{aligned}
K & =\frac{\sum_{i} k_{i}}{N} \\
& =\frac{1}{N} \sum_{i} \sum_{j} \hat{w}_{i j} \alpha_{j} s_{j},
\end{aligned}
$$

where again $\hat{w}_{i j}$ denotes the element $(i, j)$ of the matrix $\hat{\mathrm{W}}$ defined as $\hat{\mathrm{W}} \equiv[\mathrm{I}-(\mathrm{I}-\mathrm{D}) \mathrm{W}]^{-1}$. Using matrix $\mathrm{C} \equiv \hat{\mathrm{W} D}$ we can define the $1 \times N$ vector $v^{\prime}$ as

$$
v^{\prime} \equiv e^{\prime} C
$$


where $e^{\prime} \equiv[1, \ldots, 1]$, so that $v_{j}=\sum_{i} c_{i j}=\alpha_{j} \sum_{i} \hat{w}_{i j}$ and

$$
K=\frac{1}{N} \sum_{j} v_{j} s_{j}
$$

Vector $v$ can be defined as an influence vector, since each element $v_{j}$ determines the influence of signal $s_{j}$ on aggregate investment. The influence vector $v$ is related to the Bonacich (in-degree) centrality measure (Bonacich, 1987) and it is reminiscent of the influence vector described in Acemoglu et al. (2012), with the crucial difference that the impact of firm $j$ 's signal in this case also depends on $\alpha_{j}$. If the Bonacich centrality of firm $j$ (summarized by the term $\sum_{i} \hat{w}_{i j}$ ) in the observational network increases, the influence of firm $j$ 's signal will increase. On the other hand, given the observational network, increasing $\alpha_{j}$ and holding constant all $\alpha_{i \neq j}$, will increase the influence of firm $j$ 's signal. The intuition is that, if $\alpha_{j}$ is relatively high, firm $j$ 's signal will be largely reflected in its investment decision, and therefore it will have relatively higher influence on the decisions of the firms observing firm $j$. The variance of $K$ can be written as

$$
\operatorname{var}(K)=\frac{1}{N^{2}} \sum_{j} v_{j}^{2} \sigma^{2} .
$$

In the absence of social learning we have that $v_{j}=1$ for all $j$, and therefore the variance of aggregate investment is

$$
\operatorname{var}(K)=\frac{\sigma^{2}}{N}
$$

The impact of social learning on aggregate volatility is described in the following proposition:

Proposition 2. The variance of aggregate investment in the case of social learning is always greater than, or equal to, the variance of aggregate investment in the case of isolated firms, that is

$$
\frac{1}{N} \sum_{j} v_{j}^{2} \geq 1 .
$$

The proof is in Appendix A. According to Proposition 2, the case of isolated firms represents a lower bound for aggregate volatility. The intuition for this result is that social learning introduces correlation among individual decisions. We know that the variance of aggregate investment depends on $\operatorname{var}\left(\sum_{i} k_{i}\right)$, i.e., the variance of the sum of individual investment decisions. In the absence of 
social learning we have that individual decisions are independent from each other and therefore the aggregate variance is simply given by the sum of the variances of individual decisions, that is

$$
\operatorname{var}\left(\sum_{i} k_{i}\right)=\sum_{i} \operatorname{var}\left(k_{i}\right)
$$

In the presence of social learning, individual decisions are not independent and therefore aggregate variance depends also on the covariance among individual decisions, as dictated by the network structure, so that

$$
\operatorname{var}\left(\sum_{i} k_{i}\right)=\sum_{i} \operatorname{var}\left(k_{i}\right)+\sum_{i \neq j} \operatorname{cov}\left(k_{i}, k_{j}\right) .
$$

The impact of social learning is twofold. First, as shown in Proposition 1, the variance of individual decisions is lower, implying that the first term in Eq. (20) is lower when compared to the case of isolated firms in Eq. (19). Second, social learning introduces a covariance element given by the second term in Eq. (20). Proposition 2 shows that the net effect depends on the structure of the observational network and on the weights attached by each firm to the different sources of information.

In particular, if the vector $v$ has heterogeneous entries, aggregate volatility increases. The only case in which the variance of aggregate investment under social learning is equal to the case of isolation is when the signal of each firm in the economy has exactly the same influence on aggregate investment, i.e., when $v_{j}=1$ for all $j$. This scenario is verified when the network is regular, i.e., all firms have the same weighted in-degree and out-degree in the observational network, as in Fig. 1(b), and $\alpha$ is homogeneous. ${ }^{6}$ Any other case results in an influence vector with heterogeneous elements and thus aggregate volatility increases with respect to the case of isolation.

The following example illustrates the impact of heterogeneous centrality among firms.

\section{Example 3.}

Consider the two economies described in Figs. 1(a) and 1(b). Again we assume, without loss of generality, that $\alpha_{i}=\alpha \forall i$. In Section 5 we analyze the same type of economy and show that, when firms optimally set weights, qualitative results do not change. Given

\footnotetext{
${ }^{6}$ As analyzed in Section 5, if firms are able to observe directly or indirectly all other firms in the economy and set their weights optimally, they will set the weights so that the resulting network is regular, with homogeneous weighted in-degree and out-degree and homogeneous $\alpha_{i} \rightarrow 0 \forall i$.
} 
the matrices $\mathrm{C}_{\text {star }}$ and $\mathrm{C}_{\text {regular }}$ computed in Examples 1 and 2, we can compute the influence vectors associated to each network using Eq. (15):

$$
v_{\text {star }}=\left(\begin{array}{l}
2.0 \\
1.5 \\
0.5 \\
0.5 \\
0.5
\end{array}\right) \quad v_{\text {regular }}=\left(\begin{array}{l}
1.0 \\
1.0 \\
1.0 \\
1.0 \\
1.0
\end{array}\right)
$$

The influence vector is given by the sum over the columns of the matrix $\mathrm{C}$, meaning that each element $v_{i}$ is sum of the weights given by each firm to the signal $s_{i}$. The higher is $v_{i}$, the higher the centrality of firm $i$. In the star network, firm 1 is highly central since all firms look at its decision when making their own choices. Note that firm 2 is also relatively central. The reason is that the decision of firm 2 is used by firm 1. The centrality of each firm $i$, as measured by the influence vector, depends not only on the number of firms looking at firm $i$, but also on the number of firms looking at the firms who are looking at firm $i$. This means that the influence of a firm is recursively related to the influence of the firms who observe its decision (see Jackson et al., 2015). As an example, consider a modification of the star network in which, for example, firm 4 looks at firm 3 instead of firm 1. In this case the influence vector is given by $v^{\prime}=[1.83,1.42,0.75,0.5,0.5]$. Although both firms 2 and 3 are observed by one firm, the influence of firm 2 is higher than the influence of firm 3 due to the fact that firm 2 is observed by firm 1 , which is more central than firm 4 . On the contrary, in the regular network all firms have the same centrality. Using Eq. (16) we can compute the variance of aggregate production for both networks

$$
\operatorname{var}\left(K_{\text {star }}\right)=0.28 \sigma^{2} \quad \operatorname{var}\left(K_{\text {regular }}\right)=0.20 \sigma^{2}
$$

In the case of regular network aggregate variance is equal to the variance in isolation, i.e., $\sigma^{2} / 5$, meaning that the reduction in the sum of individual variances and the positive covariances in individual decisions balance each other out (see Eq. (20)). On the opposite, heterogeneity in the centrality of firms, as in the case of the star network, 
leads to an increase in aggregate volatility.

\subsection{Decay Rate of Aggregate Volatility}

The possibility of significant aggregate fluctuations originating at the micro level, i.e., at the firm or sector level, is often discarded in macroeconomics following a diversification argument. According to the latter, in an economy composed by $N$ firms (or sectors), the volatility of aggregate investment should decay at a rate $1 / \sqrt{N}$. Therefore idiosyncratic fluctuations should disappear in the aggregate as $N \rightarrow \infty$. Gabaix (2011) proves that the $1 / \sqrt{N}$ diversification argument does not apply when the firm size distribution is sufficiently fat-tailed, while Acemoglu et al. (2012) show that the argument is not valid in the presence of asymmetric input-output links between sectors.

In the following we show that, even in the presence of firms with identical size and without input-output interconnections between different sectors, the diversification argument may not hold when information is dispersed and firms try to reduce their uncertainty by learning from other firms' decisions.

Consider a sequence of economies indexed by the number of firms $N \geq 1$, with the network of informational links in each economy denoted by $\mathrm{W}_{N}$. The corresponding sequences of aggregate investment and influence vectors are denoted respectively by $\left\{K_{N}\right\}$ and $\left\{v_{N}\right\}$. Assuming that the

variance of idiosyncratic signal is independent of the size of economy $N$, e.g., $\sigma_{N}^{2}=\sigma^{2} \forall N$, we have that

$$
\operatorname{std}\left(K_{N}\right)=\sqrt{\operatorname{var}\left(K_{N}\right)}=\sigma \sqrt{\sum_{j}\left(\frac{v_{j, N}}{N}\right)^{2}} .
$$

Given two series of positive real numbers $\left\{a_{N}\right\}$ and $\left\{b_{N}\right\}$, we write $a_{N}=\Theta\left(b_{N}\right)$ if the following relationships hold simultaneously

$$
\begin{aligned}
& \lim \sup _{N \rightarrow \infty} a_{N} / b_{N}<\infty \\
& \lim \inf _{N \rightarrow \infty} a_{N} / b_{N}>0 .
\end{aligned}
$$

As shown in Acemoglu et al. (2012), when the precision of individual signals is independent of the economy's size, we have that

$$
\operatorname{std}\left(K_{N}\right)=\Theta\left(1 / N\left\|v_{N}\right\|_{2}\right)
$$


Eq. (21) implies that the volatility of aggregate production in deviation from actual demand may decay with a rate different from $1 / \sqrt{N}$, according to the properties of the network defining the patterns of social learning in the economy summarized by vector $v$.

We can use the results derived in Gabaix (2011) to characterize the decay rate of aggregate volatility when $v$ has a fat-tailed distribution. The results are summarized in the following proposition:

Proposition 3. Consider a series of economic systems indexed by $N \geq 1$. Assume that the sequence of influence vectors $v_{1}, \ldots, v_{N}$ has a power law distribution

$$
P(v>x)=a x^{-\zeta}
$$

for $x>a^{1 / \zeta}$, with exponent $\zeta \geq 1$. Then, as $N \rightarrow \infty$ aggregate volatility follows

a) $\operatorname{std}\left(\hat{K}_{N}\right) \sim \frac{v_{\zeta}}{\ln N} \sigma$ for $\zeta=1$

b) $\operatorname{std}\left(\hat{K}_{N}\right) \sim \frac{v_{\zeta}}{N^{1-1 / \zeta}} \sigma$ for $1<\zeta<2$

c) $\operatorname{std}\left(\hat{K}_{N}\right) \sim \frac{v_{\zeta}}{N^{1 / 2}} \sigma$ for $\zeta \geq 2$

where $v_{\zeta}$ is a random variable. The distribution of $v_{\zeta}$ does not depend on $N$ and $\sigma$. When $\zeta \leq 2, v_{\zeta}$ is the square root of a stable Lévy distribution with exponent $\zeta / 2$. When $\zeta>2$, $v_{\zeta}$ is a constant.

Proof. See Proposition 2 in Gabaix (2011) and proof therein. ${ }^{7}$

Proposition 3 implies that when the distribution of firms signals' influence $v$ has thin tails $(\zeta \geq 2)$, then the variance of aggregate investment decays at rate $1 / \sqrt{N}$. On the contrary, when the distribution has fat tails $(\zeta<2)$ the decay rate is much slower.

Therefore social learning may represent an additional reason for the failure of the diversification argument, depending on the topology of the informational network. In particular, a fat-tailed distribution of $v$ implies a greater heterogeneity in the influences of firms' signals, corresponding

\footnotetext{
${ }^{7}$ Defining $h=\sqrt{\sum_{j}\left(\frac{v_{j, N}}{N}\right)^{2}}$ we have that $\operatorname{std}\left(\hat{K}_{N}\right)=h \sigma$, as in Gabaix (2011). Notice that while in Gabaix (2011) $h$ is the square root of the sum of (squared) individual firms production over total GDP, i.e., the sales herfindhal of the economy, in our model $h$ is the square root of the sum of (squared) relative influence of the signal received by each firm.
} 
to the case in which many firms look at the decision of the same small number of firms. The latter group of firms have high centrality in the network and therefore the influence of their signals decays slowly as the number of firms $N$ increases.

\section{Lucas-Phelps Island Economy}

When information is dispersed and agents can observe each other's actions through a network of informational links, social learning leads to lower volatility at the level of individual actions, but at the same time it may lead to higher volatility at the aggregate level. So far we have described the consequences of dispersed information and social learning in an investment model as in Angeletos and Pavan (2004). However, the mechanism described in the previous section is general and can be applied to different frameworks. In this section we analyze the effect of social learning on individual production decisions and aggregate output in a Lucas-Phelps island economy, as in Morris and Shin (2002). Differently from Morris and Shin (2002), we do not have public information in the model, therefore we can not asses the effect on individual actions of the precision of public information released by the central bank. Our scope in using this model is different: we want to determine the effect on individual and aggregate production of a network of informational links when information is imperfect.

Consider an economy composed by $N$ islands. Supply $y_{i}^{s}$ in island $i$ of a single consumption good is given by

$$
y_{i}^{s}=b p_{i}
$$

where $b>0$ and $p_{i}$ is the price chosen in island $i$. The demand $y_{i}^{d}$ on island $i$ is given by

$$
y_{i}^{d}=c\left(\theta-p_{i}\right)
$$

where $\theta$ is the money supply and $c>0$. Market clearing implies that the optimal price decision under perfect information is

$$
p_{i}=\frac{c}{b+c} \theta
$$


When $\theta$ is unknown, each firm forms expectations on the money supply $\theta$ and sets the price

$$
p_{i}=\frac{c}{b+c} E_{i}(\theta)
$$

Assume that firms receive a private signal on the money supply as described in Eq. (3), and that $\theta$ is drawn from an improper uniform distribution. In isolation the expected money supply is

$$
E_{i}(\theta)=s_{i}
$$

and the optimal price is

$$
p_{i}=\frac{c}{b+c} s_{i}
$$

When firms are able to observe the price decision of a subset of firms in other islands, they will use this information when setting their price to increase the precision of their expectation on money supply. Similarly to Eq. (5), the price decision in the presence of social learning is:

$$
p_{i}=\alpha_{i} \beta s_{i}+\left(1-\alpha_{i}\right) \sum_{j} w_{i j} p_{j}
$$

where $\alpha_{i}$ and $w_{i j}$ have the same interpretation as in Section 2 , while $\beta \equiv c /(b+c)$. The equilibrium price decision in all islands can be written as

$$
p=[\mathrm{I}-(\mathrm{I}-\mathrm{D}) \mathrm{W}]^{-1} \mathrm{D} \beta s,
$$

where $\mathrm{W}$ is the adjacency matrix describing the network of social learning and $\mathrm{D}$ is a diagonal matrix such that $[\mathrm{D}]_{i i}=\alpha_{i} \forall i$. Firms sell at price $p_{i}$ and equilibrium production is determined by market clearing. Production on island $i$ is computed using the demand function determined by realized money supply $\theta$ and price $p_{i}$ :

$$
y_{i}=c\left(\theta-p_{i}\right)
$$

From Eq. (22) it is clear that the variance of individual production is proportional to the variance of the price decision $p_{i}$. From Proposition 1, we know that observing the price decisions of other 
firms reduces the variance of individual actions, and it is therefore beneficial to firms. Aggregate output, i.e., the GDP of the island economy, is the sum of the individual production decisions:

$$
Y=\sum_{i} y_{i}=c N \theta-c \sum_{i} p_{i}
$$

From Proposition 2, we know that given $N$, the variance of aggregate production depends on the topology of the observational network. If the network is asymmetric, aggregate volatility is higher relative to the same economy in which firms take their price decision in isolation. Finally, when the observational network is characterized by the properties in Proposition 3, then the variance of aggregate output does not decay following the law of large numbers. This finding complements the results obtained in Gabaix (2011) and Acemoglu et al. (2012) showing that aggregate fluctuations can originate at the micro level as the result of disperse information and asymmetric observational networks.

\section{Optimal Information Weighting}

DeMarzo et al. (2003) argue that persuasion bias, defined as the individual failure to properly adjust for possible repetitions when processing information, is consistent with psychological evidence, while Brandts et al. (2015) present experimental evidence showing that hat agents have difficulties in assessing correctly the information they receive. It is highly unlikely that agents in a complex network are able to objectively assess the precision of information embedded in the actions of other agents and to understand how to manage the information in order to avoid the persuasion bias. In this section we argue that even if agents were able to optimally weight information from different sources, the observational network would still play a key role in shaping aggregate fluctuations.

Optimality from an individual point of view requires the minimization of individual variance as defined in Eq. (11). As already mentioned in Section 2, optimal setting of weights $w_{i j}$ and $\alpha_{i}$ for firm $i$, given the network structure and the weights set by other firms, is a complicated problem. We will illustrate the principles driving optimality by means of four examples.

Start from an economy described by a regular network as in Fig. 1(b). A first principle of optimality for information weighting is that the weights $w_{i j}$ should reflect the objective relative 
precision of information contained in the decisions of each observed firm. In this particular case, since each firm observes only another firm in the network, the optimal weight assigned to the observed firm is equal to one. Therefore the matrix $\mathrm{W}^{*}$ of optimal weights $w_{i j}^{*}$ in this case is given by

$$
\mathrm{W}_{\text {regular }}^{*}=\left(\begin{array}{ccccc}
0 & 1 & 0 & 0 & 0 \\
0 & 0 & 1 & 0 & 0 \\
0 & 0 & 0 & 1 & 0 \\
0 & 0 & 0 & 0 & 1 \\
1 & 0 & 0 & 0 & 0
\end{array}\right) .
$$

Given the structure of the network, each firm observes directly or indirectly the decision of all other firms in the network. Therefore, for each firm $i$, the information conveyed by the network already includes its own signal $s_{i}$. Consider for example firm 1 who is observing the decision of firm 2 , which in turn observes the decision of firm 3 and so on. The structure of the network implies that firm 1 is observing indirectly firm 5, which is in turn observing firm 1 itself. Thus, the information contained in the signal of firm 1 is included in firm 1's decision both directly, with weight $\alpha_{1}$, and indirectly, with weight $1-\alpha_{1}$, by observing the decision of firm 2 . For each $\alpha_{i}>0$, we have that firm $i$ suffers from persuasion bias, in the sense that firm $i$ fails to account for the repetition of the information contained in $s_{i}$. A second principle of optimality is that, if the information conveyed by the individual signal $s_{i}$ is already present in its observational network, each firm $i$ should set $\alpha_{i}=\alpha^{*} \rightarrow 0$ to minimize the persuasion bias. ${ }^{8}$ Moreover, let $\mathrm{D}^{*}$ denote the diagonal matrix such that $\left[\mathrm{D}^{*}\right]_{i i}=\alpha^{*}, \forall i \in[1, N]$. In this case we have that each entry of the matrix $\mathrm{C}^{*} \equiv\left[\mathrm{I}-\left(\mathrm{I}-\mathrm{D}^{*}\right) \mathrm{W}^{*}\right]^{-1} \mathrm{D}^{*}$, describing equilibrium mapping of signals into decisions as per Eq. (8), is positive and equal to $1 / N$.

$$
\mathrm{C}_{\text {regular }}^{*}=\left(\begin{array}{lllll}
1 / 5 & 1 / 5 & 1 / 5 & 1 / 5 & 1 / 5 \\
1 / 5 & 1 / 5 & 1 / 5 & 1 / 5 & 1 / 5 \\
1 / 5 & 1 / 5 & 1 / 5 & 1 / 5 & 1 / 5 \\
1 / 5 & 1 / 5 & 1 / 5 & 1 / 5 & 1 / 5 \\
1 / 5 & 1 / 5 & 1 / 5 & 1 / 5 & 1 / 5
\end{array}\right)
$$

In the presence of a regular network, agents are able to set $\left\{\alpha_{i}, w_{i j}\right\}$ so that in equilibrium all signals receive a weight equal to their objective relative precision, equal to $1 / N$ given the assumption of

\footnotetext{
${ }^{8}$ Notice in fact that $\alpha^{*}$ should be strictly positive, otherwise if all firms set $\alpha_{i}=0$ there would be no information in the network (see also Lemma 1).
} 
homogeneous variance of individual signals. In general, if the network topology allows the agents to observe directly or indirectly all the signals in the economy, there exists a configuration of weights leading to an efficient use of the information by all agents.

Consider the network described by Fig. 2(a). This network is similar to the regular network, with the difference that firm 5 is linked both to firm 1 and firm 2 . If firm 5 sets both $w_{51}>0$ and $w_{52}>0$, the signal of firm 2 enters its individual decision both indirectly through the observation of firm 1's action, and directly through the observation of firm 2's decision, implying thus persuasion bias. This in turn affects the whole network, and implies an influence vector with heterogeneous elements. If firm 5 realizes that it is suffering from persuasion bias, and knows the structure of the network, it can correct the bias by setting $w_{51}=1$ and $w_{52}=0$ reducing the network in Fig. 2(a) to a regular network as in Fig. 1(b). A third principle of optimality is hence that observational links conveying no additional information are redundant. In this last example, the heterogeneous

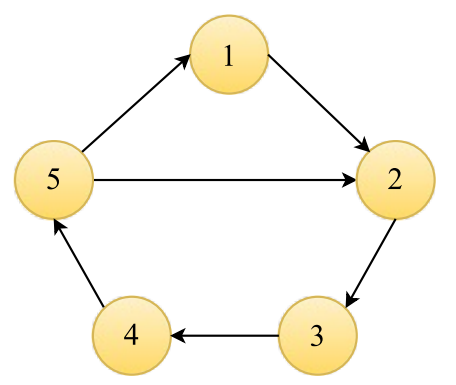

(a) Modified Regular network.

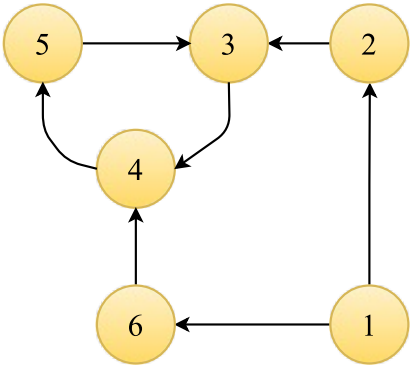

(b) Asymmetric network.

Figure 2: Different configurations of the economy.

network is reduced to a regular network by the optimizing behavior of firms. This can happen only in the special case in which the network topology allows all firms to observe, directly or indirectly, all other firms.

Consider next an economy described by a star network as in Fig. 1(a). Each firm is observing the decision of only another firm in the network, implying therefore that the optimal weight assigned 
to the observed firm is equal to one. The matrix $\mathrm{W}^{*}$ of optimal weights $w_{i j}^{*}$ is in this case given by

$$
\mathrm{W}_{\text {star }}^{*}=\left(\begin{array}{ccccc}
0 & 1 & 0 & 0 & 0 \\
1 & 0 & 0 & 0 & 0 \\
1 & 0 & 0 & 0 & 0 \\
1 & 0 & 0 & 0 & 0 \\
1 & 0 & 0 & 0 & 0
\end{array}\right)
$$

Notice that for both firms 1 and 2 the information contained in their private signal also appears in the information coming from the network. Therefore, as we showed in the previous example, in order to eliminate the persuasion bias it is optimal for firms 1 and 2 to set $\alpha_{1}^{*}=\alpha_{2}^{*} \rightarrow 0$. The private signals of firms 3, 4 and 5, are instead not present in their respective observational networks. Minimization of individual variance implies that the equilibrium contribution of signals $s_{3}, s_{4}, s_{5}$ to the decisions of firms 3,4 , and 5 respectively, should be equal to the inverse of the total number of signals affecting the equilibrium investment decisions of these firms (due to the assumption of equal signal's precisions). A fourth principle of optimality is in fact that, if firm $i$ 's private signal is not present in its observational network, this firm should set $\alpha_{i}$ in order to reflect the objective relative precision of the signals entering its equilibrium decisions. In other words, given the hypothesis of homogeneous signal variance, a signal $s_{i}$ not present in the informational network of firm $i$ should receive a weight $\alpha_{i}=1 / \sum_{j} \mathbb{I}_{c_{i j}>0}$, where the denominator gives the number of positive entries of the $i$-th row of the equilibrium matrix C. Given that firms 3, 4, and 5 observe, directly and indirectly their own signal on top of the signals of firms 1 and 2 , we have that $\alpha_{3}^{*}=\alpha_{4}^{*}=\alpha_{5}^{*}=1 / 3$. In this case the matrix $\mathrm{C}^{*}$ is given by

$$
\mathrm{C}_{\text {star }}^{*}=\left(\begin{array}{ccccc}
1 / 2 & 1 / 2 & 0 & 0 & 0 \\
1 / 2 & 1 / 2 & 0 & 0 & 0 \\
1 / 3 & 1 / 3 & 1 / 3 & 0 & 0 \\
1 / 3 & 1 / 3 & 0 & 1 / 3 & 0 \\
1 / 3 & 1 / 3 & 0 & 0 & 1 / 3
\end{array}\right)
$$

In this case each firm is able to use efficiently the information they receive. Nevertheless, the aggregate volatility is greater than the case of isolated firms. This is easy to observe by using Proposition 2 and noting that the influence vector associated to $\mathrm{C}_{\text {star }}^{*}$ is heterogeneous and equal to $v^{\prime}=[2,2,1 / 3,1 / 3,1 / 3]$. Optimality in information weighting does not remove the impact of 
asymmetric network structures.

The next example considers a case in which the network structure does not allow the agents to set efficiently the weights, i.e., individual optimality does not lead to homogeneous individual equilibrium weights. Consider the network represented in Fig. 2(b). Following the previous arguments, we know that the optimal weights assigned to the outgoing links of firms $2,3,4,5,6$ are equal to 1. Considering that firm 1 observe firms 2 and 6, and noting that the actions of firms 2 and 6 convey the same amount of information, since both firms observe their own signal and the decision of the same set of firms, it easy to conclude that $w_{12}^{*}=w_{16}^{*}=1 / 2 .{ }^{9}$ The optimal observational matrix is therefore:

$$
\mathrm{W}_{a s y}^{*}=\left(\begin{array}{cccccc}
0 & 0.5 & 0 & 0 & 0 & 0.5 \\
0 & 0 & 1 & 0 & 0 & 0 \\
0 & 0 & 0 & 1 & 0 & 0 \\
0 & 0 & 0 & 0 & 1 & 0 \\
0 & 0 & 1 & 0 & 0 & 0 \\
0 & 0 & 0 & 1 & 0 & 0
\end{array}\right) .
$$

Firms 3,4,5 observe their signal both directly and indirectly through their observational network, implying that the optimal weight on their own signal should tend to zero to avoid the persuasion bias, i.e., $\alpha_{3}^{*}=\alpha_{4}^{*}=\alpha_{5}^{*} \rightarrow 0$. Firms 2 and 6 do not observe their own signal through the network. Exactly as in the previous example, these firms must set the weights on their signal so that the impact of each signal affecting their equilibrium individual decisions is homogeneous as prescribed in the fourth principle mentioned above, and equal to the inverse of the total number of signal observed in equilibrium. In this particular case $\alpha_{2}^{*}=\alpha_{6}^{*}=1 / 4$. The problem of firm 1 is slightly different. By observing the decisions of both firm 2 and 6 , firm 1 can not avoid persuasion bias. The actions of firm 2 and firm 6 convey, beside the information from their own signals, the information reflected the actions of firms 3,4,5. Persuasion bias, i.e., using twice the information of firms $3,4,5$, is unavoidable. ${ }^{10}$ Given the optimal weights set by other firms, we can compute numerically the

\footnotetext{
${ }^{9}$ Notice that the actions of firms 2 and 6 convey the same amount of information but not exactly the same information in terms of signals, therefore none of the observational links is redundant.

${ }^{10}$ Notice also that setting either $w_{12}$ or $w_{16}$ equal to zero would result in the loss of the information contained in one of the signals, leading therefore to a suboptimal choice.
} 
optimal choice of $\alpha_{1}^{*}=0.1795$. In this case the matrix $\mathrm{C}^{*}$ is given by

$$
\mathrm{C}_{a s y}^{*}=\left(\begin{array}{cccccc}
0.1795 & 0.1026 & 0.2051 & 0.2051 & 0.2051 & 0.1026 \\
0 & 0.2500 & 0.2500 & 0.2500 & 0.2500 & 0 \\
0 & 0 & 0.3333 & 0.3333 & 0.3333 & 0 \\
0 & 0 & 0.3333 & 0.3333 & 0.3333 & 0 \\
0 & 0 & 0.2500 & 0.2500 & 0.2500 & 0.2500
\end{array}\right)
$$

Individual rationality leads firms $2,3,4,5,6$ to optimally aggregate the available information. On the contrary, optimal weights setting in the case of firm 1 does not allow to efficiently aggregate its available information. The reason is that firm 1's optimal choice of weights does not allow to avoid double-counting part of the information in the network. Moreover, the influence vector associated to the matrix $\mathrm{C}_{a s y}^{*}$ is heterogeneous, leading to an increase in aggregate volatility with respect to the case of isolation.

The network structure has important consequences both at the individual and the aggregate level, also when optimally weight information, having perfect information about both the topology of the network and the weights chosen by the other agents. Individual rationality can eliminate the amplification effect of aggregate volatility due to social learning only if all firms are able to observe directly and indirectly all other firms, and the topology of the network allows them to avoid possible repetitions in the information they receive. As soon as the network displays some asymmetries, dispersed information and observation learning will affect aggregate volatility also when agents optimally weight available information.

\section{Conclusions}

The behavior of peers, friends or in general other members of a social or economic group, represents a valuable source of information for the homo oeconomicus. Observation of others' behavior is deeply rooted in human nature as a consequence of the adaptation to a complex environment, where it is difficult to collect and process all available information. This paper shows that this micro-behavior, which we have called social learning, can have relevant consequences at the aggregate level. The aggregate effect of social learning depends on the topology of the network describing the links between the agents. We proved that the network structure can explain, at least in part, aggregate 
fluctuations.

If the network is homogeneous, in the sense that all firms have the same influence in equilibrium, then aggregate fluctuations with social learning coincide with aggregate fluctuations when firms are isolated. For any other network configuration, social learning leads to an increase in aggregate volatility. Aggregate variance is positively related to the concentration of influence in the network.

Moreover, we show that the diversification argument does not always apply in the presence of social learning. If the influence vector is sufficiently asymmetric, i.e., there exist few very influential firms in the network, then aggregate variance does not decay at a rate equal to $1 / \sqrt{N}$ as $N \rightarrow \infty$. This result complements the findings of Gabaix (2011), who shows that the diversification argument does not hold when firms' size distribution is sufficiently fat-tailed, and Acemoglu et al. (2012) who show that the law of large number argument breaks down in the presence of asymmetric inputoutput links between productive sectors.

An important question regards the empirical relevance of social learning. From a qualitatively point of view, the impact of social learning crucially depends, as argued above, on the topology of the observational network. In practice the network structure characterizing how agents learn from each other is difficult to observe. It is possible to look for proxies using observable social-economic networks, or propose a network formation model (see e.g., Bala and Goyal, 2000) in order to analyze possible theoretical configurations of the network. In general, in the case of firms, we believe that it is highly plausible that the influence of a firm in the observational network is strongly and positively correlated to the importance of the firm with respect to some relevant economic variables. For example bigger firms might be easier (or cheaper) to observe than smaller firms, and given the strongly skewed distribution of firms' size (Axtell, 2001; Gaffeo et al., 2003), it is plausible to think of an asymmetrical observational network. Similarly, assuming that firms observe the decisions of downstream or upstream partners, one could describe the topology of the observational network using the topology of the input-output network. Given the empirical properties of input-output networks, this would imply an asymmetrical observational network. Moreover, Bikhchandani et al. $(1992,1998)$ argue in favor of the presence of fashion leaders, i.e., "expert" agents observed by many other agents, and Gilbert and Lieberman (1987) show that "smaller firms tend to imitate the investment activity of others". Therefore, we conclude that it is highly plausible that observational networks are asymmetric, and consequently that dispersed information and social learning play an 
important role in generating aggregate fluctuations.

\section{Appendix A Proofs}

\section{A.1 Proof of Lemma 1}

Proof. Iterating Eq. (7) we get

$$
k_{\tau}=\sum_{z=1}^{\tau-1}[(\mathrm{I}-\mathrm{D}) \mathrm{W}]^{z} \mathrm{D} s+[(\mathrm{I}-\mathrm{D}) \mathrm{W}]^{\tau} k_{0}
$$

Define $\mathrm{A} \equiv(\mathrm{I}-\mathrm{D}) \mathrm{W}$ and notice that $\lim _{\tau \rightarrow \infty} \mathrm{A}^{\tau}=0$ and $\sum_{z=0}^{\infty} \mathrm{A}^{z}=[\mathrm{I}-\mathrm{A}]^{-1}$ when the spectral radius of $\mathrm{A}$, defined as $\rho(\mathrm{A})=\max _{1 \leq i \leq N}\left|\lambda_{i}\right|$ where $\lambda_{i}$ is the $i$-th eigenvalue of $\mathrm{A}$, is strictly smaller than one. Then notice that

$$
\|A\|_{\infty}=\max \left\{\sum_{j=1}^{N}\left|\left(1-\alpha_{i}\right) w_{i j}\right| \mid 1 \leq i \leq N\right\}=\max \left\{\left|\left(1-\alpha_{i}\right)\right| \mid 1 \leq i \leq N\right\}
$$

given that matrix $\mathrm{W}$ is stochastic. Moreover, for a generic eigenvector-eigenvalue pair $(x, \lambda)$ with $x \neq 0$, we have that $\lambda x=\mathrm{A} x$ and therefore

$$
\|\lambda x\|_{\infty}=|\lambda|\|x\|_{\infty}=\|\mathrm{A} x\|_{\infty} \leq\|\mathrm{A}\|_{\infty}\|x\|_{\infty} \Rightarrow|\lambda| \leq\|\mathrm{A}\|_{\infty},
$$

where the inequality follows from the submultiplicativity property of the matrix norm.

In case a) of Lemma 1, i.e., when $0<\alpha_{i} \leq 1 \forall i \in[1, N]$, we have that $\|A\|_{\infty}<1$ implying that $\rho(\mathrm{A})<1$.

In order to prove the result in case b) of Lemma 1, we start by proving that if A is irreducible and at least one $\alpha_{i}>0$, then it must be that $\rho(A)<1$. Notice that when at least one $\alpha_{i}>0$, then matrix $\mathrm{A}$ is substochastic. Denoting by $e^{\prime}=[1 \ldots 1]$, this implies that $\mathrm{A} e \leq e$ and $\mathrm{A} e \neq e$. When matrix $\mathrm{A}$ is irreducible, from the Perron-Frobenius theorem it follows that $\rho(\mathrm{A})=1$ would imply $\mathrm{A} e=e$, which is impossible by construction. Therefore $\rho(\mathrm{A})<1$ follows from the result $\rho(A) \leq\|\mathrm{A}\|_{\infty}$ derived above.

Let's now consider the case in which $\mathrm{A}$ is reducible. In general, if $0 \leq \alpha_{i}<1$, the reducibility 
of matrix A follows from the reducibility of matrix W. If instead $\alpha_{i}=1$ for some agents $i \in[1, N]$, then matrix $\mathrm{A}$ is reducible even if matrix $\mathrm{W}$ is irreducible. In what follows we define conditions such that $\rho(A)<1$ when $\mathrm{A}$ is reducible. If $\mathrm{A}$ is reducible, then following Meyer (2000, page 694), it is possible to write matrix $\mathrm{A}$ in the canonical form for reducible matrices

$$
\mathrm{A} \sim\left(\begin{array}{cccc|cccc}
\mathrm{A}_{11} & \mathrm{~A}_{12} & \cdots & \mathrm{A}_{1 r} & \mathrm{~A}_{1, r+1} & \mathrm{~A}_{1, r+2} & \cdots & \mathrm{A}_{1, m} \\
0 & \mathrm{~A}_{22} & \cdots & \mathrm{A}_{2 r} & \mathrm{~A}_{2, r+1} & \mathrm{~A}_{2, r+2} & \cdots & \mathrm{A}_{2, m} \\
\vdots & & \ddots & \vdots & \vdots & \vdots & \cdots & \vdots \\
0 & 0 & \cdots & \mathrm{A}_{r r} & \mathrm{~A}_{r, r+1} & \mathrm{~A}_{r, r+2} & \cdots & \mathrm{A}_{r, m} \\
\hline 0 & 0 & \cdots & 0 & \mathrm{~A}_{r+1, r+1} & 0 & \cdots & 0 \\
0 & 0 & \cdots & 0 & 0 & \mathrm{~A}_{r+2, r+2} & \cdots & 0 \\
\vdots & & \cdots & \vdots & \vdots & \vdots & \ddots & \vdots \\
0 & 0 & \cdots & 0 & 0 & 0 & \cdots & \mathrm{A}_{m, m}
\end{array}\right)
$$

where each $\mathrm{A}_{11}, \ldots, \mathrm{A}_{r r}$ is either irreducible or $[0]_{1 \times 1}$, and $\mathrm{A}_{r+1, r+1}, \ldots \mathrm{A}_{m m}$ are irreducible. As noted in Meyer (2000), the effect of such a symmetric permutation is simply to relabel the nodes in the original network. Therefore a generic $\mathrm{A}_{s z}$ denotes the sub-network describing the connections from agents in rows $s$ to agents in columns $z$. Define $\alpha(s)$ as the vector containing the $\alpha_{i}$ set by each agent $i$ belonging to the set described by rows $s$.

As a first step, consider the matrices $\mathrm{A}_{k k}$ for $k=1,2, \ldots, r$ and observe that $\rho\left(\mathrm{A}_{k k}\right)<1$ for each $k=1,2, \ldots, r$, for any possible value of entry $\alpha_{i}$ in vector $\alpha(k)$. This is certainly true when $\mathrm{A}_{k k}=[0]_{1 \times 1}$, so consider the case in which $\mathrm{A}_{k k}$ is irreducible and notice that $A_{k k}$ is substochastic by construction because there must be blocks $\mathrm{A}_{k j}, j \neq k$ that have nonzero entries. From the previous result we know that irreducible substochastic matrices are characterized by a spectral radius strictly smaller than one.

Consider now the matrices $\mathrm{A}_{k k}$ for $k=r+1, \ldots, m$, which refer to the minimally closed groups relative to $\mathrm{A}$. These matrices are substochastic if and only if at least one $\alpha_{i}$ in vector $\alpha(k)$ is positive. Once again, these irreducible stochastic matrices are characterized by a spectral radius strictly smaller than one.

Therefore, when at least one agent $i$ in each minimally closed group of A sets $\alpha_{i}>0$, we conclude that $\rho(\mathrm{A})<1$. 
In other words, for equilibrium in Eq. (8) to exist, each agent must receive at least one signal directly and/or indirectly. If $\alpha_{i}>0 \forall i$, this condition is satisfied for any possible network topology. If instead $\alpha_{i}=0$ for some agent $i$, then for equilibrium in Eq. (8) to exist, at least one agent $i$ in any minimally closed group must have $\alpha_{i}>0$ guaranteeing that all agents in the network are reached by at least one signal.

\section{A.2 Proof of Proposition 1}

Proof. Before proceeding with the proof of Proposition 1, we describe an important property of the matrix $\mathrm{C} \equiv[\mathrm{I}-(\mathrm{I}-\mathrm{D}) \mathrm{W}]^{-1} \mathrm{D}$, which maps private signals into equilibrium investment decisions according to Eq. (8), in the following lemma.

Lemma 2. Matrix $C$ is a stochastic matrix, i.e., $\sum_{j} \hat{w}_{i j} \alpha_{j}=1 \forall i$.

Proof of Lemma 2. Define the vector $e^{\prime}=[1 \ldots 1]$. Proving that $\sum_{j} \hat{w}_{i j} \alpha_{j}=1 \forall i$ is equivalent to prove that $\mathrm{C} e=e$ or equivalently that $\mathrm{C}^{-1} e=e$, since $\mathrm{C}$ is invertible. Start from

$$
\mathrm{C}=[\mathrm{I}-(\mathrm{I}-\mathrm{D}) \mathrm{W}]^{-1} \mathrm{D},
$$

and pre-multiply both sides by $\mathrm{C}^{-1}$ to get

$$
\mathrm{I}=\mathrm{C}^{-1}[\mathrm{I}-(\mathrm{I}-\mathrm{D}) \mathrm{W}]^{-1} \mathrm{D}
$$

Post-multiplying by $\mathrm{D}^{-1}$

$$
\mathrm{D}^{-1}=\mathrm{C}^{-1}[\mathrm{I}-(\mathrm{I}-\mathrm{D}) \mathrm{W}]^{-1},
$$

and by $[\mathrm{I}-(\mathrm{I}-\mathrm{D}) \mathrm{W}]$ we get

$$
\mathrm{D}^{-1}[\mathrm{I}-(\mathrm{I}-\mathrm{D}) \mathrm{W}]=\mathrm{C}^{-1}
$$


Post-multiplying both sides by $e$, we have

$$
\begin{aligned}
\mathrm{C}^{-1} e & =\mathrm{D}^{-1}[\mathrm{I}-(\mathrm{I}-\mathrm{D}) \mathrm{W}] e \\
\mathrm{C}^{-1} e & =\mathrm{D}^{-1}[e-(\mathrm{I}-\mathrm{D}) \mathrm{W} e] \\
\mathrm{C}^{-1} e & =\mathrm{D}^{-1}[e-(\mathrm{I}-\mathrm{D}) e] \\
\mathrm{C}^{-1} e & =\mathrm{D}^{-1}[e-e+\mathrm{D} e] \\
\mathrm{C}^{-1} e & =\mathrm{D}^{-1} \mathrm{D} e \\
\mathrm{C}^{-1} e & =e,
\end{aligned}
$$

where the third equality follows from the fact that $\mathrm{W}$ is stochastic.

Having established the result in Lemma 2 we can proceed to prove Proposition 1 as follows. Eq. (13) follows from the comparison of Eqs. (11) and (12). We start by defining an M-matrix (Plemmons, 1977):

Definition. An $N \times N$ matrix $C$ that can be expressed in the form $C=s I-A$, where $a_{i j} \geq 0$ is the (ij)-th element of matrix $A, 1 \leq i, j \leq N$ and $s \geq \rho(A)$, the maximum of the moduli of the eigenvalues of $A$, is called an M-matrix.

It is straightforward to show that matrix $\mathrm{I}-(\mathrm{I}-\mathrm{D}) \mathrm{W}$ is an M-matrix. Define $s=1$ and A $=$ $(\mathrm{I}-\mathrm{D}) \mathrm{W}$. By construction we know that $a_{i j} \geq 0$, while we showed in the proof of Lemma 1 that $\rho(\mathrm{A}) \leq 1$.

Since I - (I - D)W is an M-matrix, we know that it is inverse-positive (Plemmons, 1977), i.e., each element $\hat{w}_{i j}$ of $\hat{\mathrm{W}}=[\mathrm{I}-(\mathrm{I}-\mathrm{D}) \mathrm{W}]^{-1}$ is non-negative. From Lemma 2 we know that $\sum_{j} \hat{w}_{i j} \alpha_{j}=1$ and therefore, given that $0<\alpha_{j} \leq 1 \forall j$, we have that $0 \leq \hat{w}_{i j} \alpha_{j}<1 \forall j$. Therefore, defining $f(x)=x^{2}$, we have that

$$
\sum_{j} f\left(\hat{w}_{i j} \alpha_{j}\right) \leq f\left(\sum_{j} \hat{w}_{i j} \alpha_{j}\right)=1
$$

where the inequality follows from the fact that $f$ is a superadditive function for non-negative real numbers. The only case in which the above expression holds as an equality is when there is no social learning, i.e., when $\mathrm{W}$ is a zero matrix (meaning that $\hat{\mathrm{W}}$ is an identity matrix) and $\alpha_{j}=1 \forall j$. 


\section{A.3 Proof of Proposition 2}

Proof. Eq. (18) follows from the comparison of Eqs. (16) and (17). We can rewrite Eq. (18) as

$$
\sum_{j} v_{j}^{2} \geq N \Rightarrow\|v\|_{2} \geq \sqrt{N}
$$

and prove it using the Cauchy-Schwarz inequality. In fact, noticing from the results in Lemma 2 that $\sum_{j} v_{j}=N$, we can write

$$
\begin{aligned}
\left(\sum_{j} v_{j}^{2}\right) \cdot N & \geq\left(\sum_{j} v_{j}\right)^{2} \\
\|v\|_{2} \sqrt{N} & \geq N \\
\|v\|_{2} & \geq \sqrt{N}
\end{aligned}
$$

\section{Appendix B Observing Private Signals}

In this appendix we assume that agents observe other agents' private signals, rather than other agents' actions, and show that, mutatis mutandis, all the propositions in Section 3 hold. Assume that the framework is the same as the one described in Section 2, with the only difference that firms observe the private signal of a subset of other firms. The investment decision of firm $i$ can be rewritten as:

$$
k_{i}=\sum_{j} w_{i j} s_{j}
$$

where the $w_{i j}$ is the element $(i, j)$ of the stochastic matrix $\mathrm{W}$, determining the weight assigned by agent $i$ to the signal of agent $j$, and $s_{j}$ is the private signal received by agent $j$. Matrix W is an $N \times N$ adjacency matrix describing the topology of the observational network, where $N$ is the total number of agents in the economy and each element $w_{i j}>0$ indicates the existence of an informational link from firm $i$ to firm $j$, i.e., firm $i$ can observe the private signal of firm $j$. Matrix W can be asymmetric, and differently from the model described in Section 2 we assume that $w_{i i}>0 \forall i$, meaning that, quite naturally, firms take into account their own signal when 
making investment plans. ${ }^{11}$ The investment decision in Eq. (23) is therefore a weighted average of the signals observed by agent $i$, where $w_{i j}>0$ can be interpreted as a subjective evaluation of the precision of the agent $j$ 's private signal by agent $i$. The investment decisions in the economy can be written as:

$$
k=\mathrm{W} s,
$$

where $k$ is the $N \times 1$ vector of investment decision and $s$ is the $N \times 1$ vector if signals. The aggregate investment, normalized by the number of agents is:

$$
K=\frac{1}{N} \sum_{i} k_{i}
$$

As in the model described in Section 2, all private signals are independent and have the same variance $\sigma^{2}$.

Individual volatility. The variance of the individual investment decision is

$$
\operatorname{var}\left(k_{i}\right)=\sum_{j} w_{i j}^{2} \sigma^{2}
$$

By assumption $\sum_{j} w_{i j}=1$, therefore $\left(\sum_{j} w_{i j}\right)^{2}=1$. We can therefore reproduce the proof of Proposition 1 by defining $f(x)=x^{2}, \lambda_{j}=w_{i j} / \sum_{j} w_{i j}$ and $x=\sum_{j} w_{i j}$, and writing

$$
\sum_{j} w_{i j}^{2}=\sum_{j} f\left(w_{i j}\right)=\sum_{j} f\left(\lambda_{j} x\right) \leq \sum_{j} \lambda_{j} f(x)=f(x)=f\left(\sum_{j} w_{i j}\right)=1,
$$

where the inequality follows from the fact that $f$ is convex and $f(0)=0$. The only case in which the above expression holds as an equality is when there is only one element on the row $w_{i}$. different from zero. Since we have assumed that $w_{i i}>0$, this is the case in which firm $i$ does not observe other firms in the economy and $w_{i i}=1$. If firm $i$ observes at least another signal, beside its own, then $\sum_{j} w_{i j}^{2}<1$

\footnotetext{
${ }^{11}$ We remark that assuming $w_{i i} \geq 0$ does not change our results. We consider the case $w_{i i}>0$ in order to facilitate comparison with the case of isolation.
} 
Aggregate volatility. The variance of aggregate investment is:

$$
\operatorname{var}(K)=\frac{1}{N^{2}} \operatorname{var}\left(\sum_{i} k_{i}\right),
$$

from which

$$
\operatorname{var}(K)=\frac{1}{N^{2}} \operatorname{var}\left(\sum_{i} \sum_{j} w_{i j} s_{j}\right) .
$$

Let's define the influence of firm $j$ as $v_{j} \equiv \sum_{i} w_{i j}$, which corresponds to its weighted in-degree. In this setting, the influence of firm $j$ depends directly on the weight given by other firms in the economy to the signal received by agent $j$. We can rewrite the variance of aggregate investment as:

$$
\operatorname{var}(K)=\frac{1}{N^{2}} \operatorname{var}\left(\sum_{j} v_{j} s_{j}\right) .
$$

Using the fact that signals are independent, we can rewrite the variance of aggregate investment as

$$
\operatorname{var}(K)=\frac{\sigma^{2}}{N^{2}} \sum_{j} v_{j}^{2}
$$

In the case of isolated firms, the elements of the influence vectors are homogeneous and equal to 1 , so that the term $\sum_{j} v_{j}^{2}=N$ and the variance of aggregate investment is $\operatorname{var}(K)=\frac{\sigma^{2}}{N}$. In Proposition 2 we show that the variance of aggregate investment is always equal to, or greater than, the volatility in isolation, i.e.,

$$
\frac{\sigma^{2}}{N^{2}} \sum_{j} v_{j}^{2} \geq \frac{\sigma^{2}}{N}
$$

The proof of Proposition 2 can be reprised in the current setting, simply by noticing that $\sum_{j} v_{j}=N$. This property comes from the assumption that $\mathrm{W}$ is a stochastic matrix, implying that the sum of all the elements of $\mathrm{W}$ is equal to $N$. Since $v_{j}$ is the sum of the elements of column $j$, it follows that $\sum_{j} v_{j}=N$.

Similarly we can translate the conditions on the distribution of $v$ identified in Proposition 3 directly in the current setting. If the distribution of $v_{j}$ has fat tails, i.e., few agents are observed by many other agents in the economy, then the decay rate of aggregate volatility does not follow the law of large numbers. 
The difference between the case in which the agents observe the actions of other agents, and the case in which the agents observe directly the private signal of other agents, boils down to the different definition of the influence vector $v$.

\section{References}

Acemoglu, D., Carvalho, V. M., Ozdaglar, A., and Tahbaz-Salehi, A. (2012). The network origins of aggregate fluctuations. Econometrica, 80(5):1977-2016.

Acemoglu, D., Dahleh, M. A., Lobel, I., and Ozdaglar, A. (2011). Bayesian learning in social networks. The Review of Economic Studies, 78(4):1201-1236.

Angeletos, G. M., Iovino, L., and La'O, J. (2016). Real rigidity, nominal rigidity, and the social value of information. American Economic Review, 106(1):200-227.

Angeletos, G. M. and La'O, J. (2013). Sentiments. Econometrica, 81(2):739-779.

Angeletos, G. M. and Pavan, A. (2004). Transparency of information and coordination in economies with investment complementarities. American Economic Review, 94(2):91-98.

Angeletos, G. M. and Pavan, A. (2007). Efficient use of information and social value of information. Econometrica, 75(4):1103-1142.

Apesteguia, J., Huck, S., and Oechssler, J. (2007). Imitation theory and experimental evidence. Journal of Economic Theory, 136(1):217-235.

Asch, S. E. (1961). Effects of group pressure upon the modification and distortion of judgments. In Henle, M., editor, Documents of Gestalt Psychology, pages 222-236. University of California Press, Berkeley and Los Angeles, California, USA.

Axtell, R. L. (2001). Zipf distribution of us firm sizes. Science, 293(5536):1818-1820.

Bak, P., Chen, K., Scheinkman, J., and Woodford, M. (1993). Aggregate fluctuations from independent sectoral shocks: self-organized criticality in a model of production and inventory dynamics. Ricerche Economiche, 47(1):3-30. 
Bala, V. and Goyal, S. (1998). Learning from neighbours. The Review of Economic Studies, 65(3):595-621.

Bala, V. and Goyal, S. (2000). A noncooperative model of network formation. Econometrica, 68(5):1181-1229.

Bandura, A. and McClelland, D. C. (1977). Social learning theory. Prentice-Hall Englewood Cliffs, NJ.

Banerjee, A. V. (1992). A simple model of herd behavior. The Quarterly Journal of Economics, pages $797-817$.

Benhabib, J., Wang, P., and Wen, Y. (2015). Sentiments and aggregate demand fluctuations. Econometrica, 83(2):549-585.

Bikhchandani, S., Hirshleifer, D., and Welch, I. (1992). A theory of fads, fashion, custom, and cultural change as informational cascades. Journal of political Economy, pages 992-1026.

Bikhchandani, S., Hirshleifer, D., and Welch, I. (1998). Learning from the behavior of others: Conformity, fads, and informational cascades. The Journal of Economic Perspectives, pages $151-170$.

Bonacich, P. (1987). Power and centrality: A family of measures. American journal of sociology, pages $1170-1182$.

Brandts, J., Giritligil, A. E., and Weber, R. A. (2015). An experimental study of persuasion bias and social influence in networks. European Economic Review, 80:214 - 229.

Chahrour, R. and Gaballo, G. (2015). On the nature and stability of sentiments. Technical report, Boston College Department of Economics.

Colombo, L., Femminis, G., and Pavan, A. (2014). Information acquisition and welfare. The Review of Economic Studies, 81(4):1438-1483.

DeGroot, M. H. (1974). Reaching a consensus. Journal of the American Statistical Association, $69(345): 118-121$. 
DeMarzo, P. M., Vayanos, D., and Zwiebel, J. (2003). Persuasion bias, social influence, and unidimensional opinions. The Quarterly Journal of Economics, 118(3):909 - 928.

Dupor, B. (1999). Aggregation and irrelevance in multi-sector models. Journal of Monetary Economics, 43(2):391-409.

Durlauf, S. N. (1993). Nonergodic economic growth. The Review of Economic Studies, 60(2):349366.

Ellison, G. and Fudenberg, D. (1993). Rules of thumb for social learning. Journal of Political Economy, 101(4):612-643.

Ellison, G. and Fudenberg, D. (1995). Word-of-mouth communication and social learning. Quarterly Journal of Economics, 110(1):93-125.

Fernandez, G. J., Capurro, A. F., and Reboreda, J. C. (2003). Effect of group size on individual and collective vigilance in greater rheas. Ethology, 109(5):413-425.

Gabaix, X. (2011). The granular origins of aggregate fluctuations. Econometrica, 79(3):733-772.

Gaffeo, E., Gallegati, M., and Palestrini, A. (2003). On the size distribution of firms: additional evidence from the G7 countries. Physica A: Statistical Mechanics and its Applications, 324(1):117-123.

Gale, D. and Kariv, S. (2003). Bayesian learning in social networks. Games and Economic Behavior, 45(2):329-346.

Gilbert, R. J. and Lieberman, M. (1987). Investment and coordination in oligopolistic industries. The Rand Journal of Economics, pages 17-33.

Golub, B. and Jackson, M. O. (2010). Naive learning in social networks and the wisdom of crowds. American Economic Journal: Microeconomics, pages 112-149.

Henrich, J. (2015). The secret of our success: How culture is driving human evolution, domesticating our species, and making us smarter. Princeton University Press.

Horvath, M. (1998). Cyclicality and sectoral linkages: Aggregate fluctuations from independent sectoral shocks. Review of Economic Dynamics, 1(4):781-808. 
Horvath, M. (2000). Sectoral shocks and aggregate fluctuations. Journal of Monetary Economics, 45(1):69-106.

Jackson, M. O., Rogers, B. W., and Zenou, Y. (2015). The economic consequences of social network structure. Available at SSRN.

Jovanovic, B. (1987). Micro Shocks and Aggregate Risk. The Quarterly Journal of Economics, 102(2):395-409.

Keynes, J. M. (1936). The general theory of interest, employment and money. London: Macmillan.

Lahno, A. M. and Serra-Garcia, M. (2015). Peer effects in risk taking: Envy or conformity? Journal of Risk and Uncertainty, 50(1):73-95.

Lucas, R. E. (1977). Understanding business cycles. Carnegie-Rochester Conference Series on Public Policy, 5(1):7-29.

Meyer, C. D. (2000). Matrix analysis and applied linear algebra. Society for industrial and applied mathematics.

Morris, S. and Shin, H. S. (2002). Social value of public information. American Economic Review, 92(5):1521-1534.

Plemmons, R. (1977). M-matrix characterizations . i-nonsingular m-matrices. Linear Algebra and its Applications, 18(2):175 - 188.

Pulliam, H. R. (1973). On the advantages of flocking. Journal of Theoretical Biology, 38(2):419-422.

Smith, L. and Sørensen, P. (2000). Pathological outcomes of observational learning. Econometrica, 68(2):371-398. 